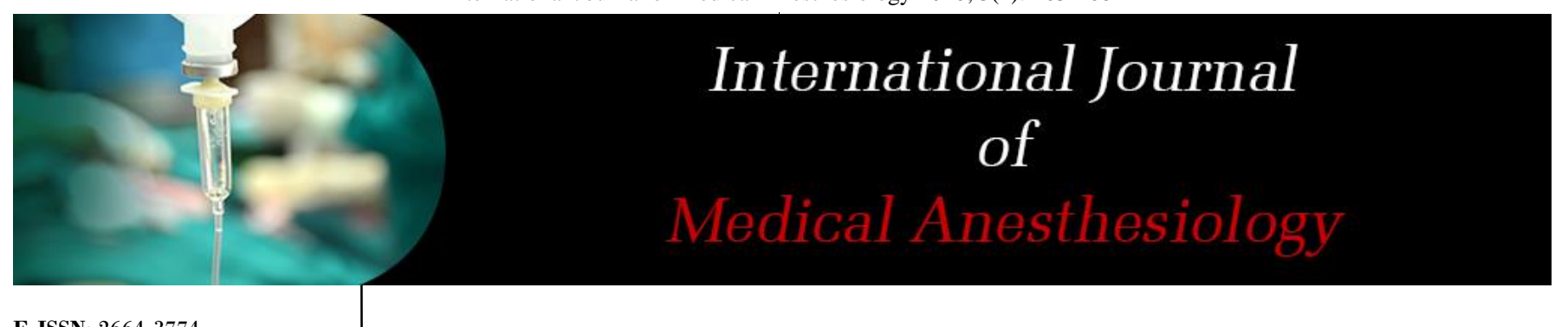

E-ISSN: 2664-3774

P-ISSN: 2664-3766

www.anesthesiologypaper.com

IJMA 2020; 3(4): 163-168

Received: 29-09-2020

Accepted: 30-10-2020

Dr. Kruthika HV

Registrar, Department of

Anaesthesia, Manipal

Hospital, Whitefield,

Bangalore, Karnataka, India

Dr. Anitha Diwakar

Consultant Anaesthesiologist,

Department of Anaesthesiology and Critical Care, Sri Sathya

Sai Institute of Higher Medical

Sciences, Bangalore,

Karnataka, India

Dr. Kolli S Chalam

Professor and Head,

Department of Anaesthesiology

and Critical Care, Sri Sathya

Sai Institute of Higher Medical

Sciences, Bangalore,

Karnataka, India

Dr. Pankaj Punetha

Consultant Anaesthesiologist, Department of Anaesthesiology and Critical Care, Sri Sathya

Sai Institute of Higher Medical

Sciences, Bangalore,

Karnataka, India

Dr. Swathi V

Resident, Indira Gandhi

Institute of Child Health,

Bangalore, Karnataka, India

Corresponding Author:

Dr. Kruthika HV

Registrar, Department of

Anaesthesia, Manipal

Hospital, Whitefield,

Bangalore, Karnataka, India

\section{A comparative study of intravenous combination of Dexmedetomidine and ketamine versus fentanyl and ketamine as sedative agents in children undergoing interventional Cardiac Catheterization procedures}

\author{
Dr. Kruthika HV, Dr. Anitha Diwakar, Dr. Kolli S Chalam, Dr. Pankaj \\ Punetha and Dr. Swathi V
}

DOI: https://doi.org/10.33545/26643766.2020.v3.i4c.181

\begin{abstract}
Fentanyl is a highly selective mu-agonist (or MOP agonist); the MOP receptor appears to be specifically involved in the mediation of analgesia. Opioids appear to exert their effects by interacting with presynaptic Gi-protein receptors, leading to hyperpolarization of the cell membrane by increasing $\mathrm{K}+$ conductance. Inhibition of adenylate cyclase, leading to reduced production of cAMP, and closure of voltage-sensitive calcium channels also occur. After institutional scientific and ethical committee approval, a written informed consent was obtained from parents. 70 paediatric patients, American Society of Anesthesiologists (ASA) physical status II and III, aged between 4 to 12 yrs with acyanotic congenital heart disease admitted for elective interventional cardiac catheterization procedures are included in this study. There is significant difference in the mean duration of sedation attained by group $\mathrm{D}$ and group $\mathrm{F}$ both statistically and clinically. The sedation time does include the procedure duration and duration from end of procedure to attainment of the steward score of 7 .
\end{abstract}

Keywords: Dexmedetomidine, Ketamine, Fentanyl

\section{Introduction}

With invention of minimally invasive procedures and use of natural orifices to identify diagnose and treat the pathologies NORA has become established body in world of anaesthesia.

NORA has increased our horizons to outside the operation theatre (OR) making us more dynamic and at the same time cautious and well read.

NORA has also come with challenges such as hostile environment, increased interdepartmental understanding. It is commonly offered to children and patients who are at increased risk of morbidity and mortality with routine surgery and anesthesia ${ }^{[1]}$.

With technological improvement in cardiac imaging and devices wider range of nonsurgical or minimally invasive options are becoming the choice of treatment.

Pediatric perioperative cardiac arrest registry shows that nearly one-third of cardiac arrests occur in children with congenital heart disease (CHD), of which $17 \%$ occur during cardiac catheterization ${ }^{[2]}$.

There by cardiac catheterization suite is a challenging environment. The isolation from our routine anesthesia resources combined with reduced functional space hinders access to the patient can make emergency resuscitation more difficult.

Propofol as a sole agent and in combination as a bolus or in TIVA have been tried in many studies.

Dexmedetomidine is used as a sole agent as well as adjuvant for sedation in many of surgical procedure like cochlear implant surgery, as well as for interventions in Cath lab in combination with ketamine. Dexmedetomidine in combination with Ketamine has provided effective sedation for cardiac catheterization in infants and children without significant effects on cardiovascular or ventilatory function ${ }^{[3]}$.

Fentanyl is also being used as a sedative agents alone or in combination in emergency department in orthopedic procedures

Midazolam is a water-soluble benzodiazepine with an imidazole ring in its structure that 
accounts for stability in aqueous solutions and rapid metabolism.

The amnestic properties of midazolam is more potent and predominant than sedative effect.

It is available as $5 \mathrm{mg}$ and $10 \mathrm{mg}$ vials. The Pka of midazolam is 6.15 , which permits the preparation of salts that are water soluble. Its known to have slow effect-site equilibration time at the brain GABA receptor levels (0 5to 5.6 minutes) compared with other drugs such as propofol and thiopental ${ }^{[4]}$.

Ketamine is a phencyclidine derivative known for its "dissociative anaesthesia," which is characterized by evidence on the EEG of dissociation between the thalamocortical and limbic systems.

Dissociative anaesthesia resembles a cataleptic state in which the eyes remain open with slow nystagmic gaze. The patient is noncommunicative, although wakefulness is observed. The patient is amnesic, and analgesia is intense.

Fentanyl is a highly selective mu-agonist (or MOP agonist); the MOP receptor appears to be specifically involved in the mediation of analgesia. Opioids appear to exert their effects by interacting with presynaptic Gi-protein receptors, leading to hyperpolarization of the cell membrane by increasing $\mathrm{K}+$ conductance. Inhibition of adenylate cyclase, leading to reduced production of cAMP, and closure of voltagesensitive calcium channels also occur. The decrease in membrane excitability that results in the decrease of both pre- and post-synaptic responses ${ }^{[5]}$.

To minimize administration of multiple drugs and side effects of large dosing of a single drug and also keeping in view of goals of anaesthesia for pediatric cardiac catherisation interventions, the sedative drug combinations of dexmedetomidine with ketamine and fentanyl with ketamine were chosen for our study ${ }^{[6]}$.

There is paucity of literature comparing Dexmedetomidine and Fentanyl in combination with Ketamine as sedative agents in pediatric interventional cardiac catheterization procedures such as device closure of ASD, VSD and PDA, hence the present study was being undertaken to evaluate the efficacy of dexmedetomidine with Ketamine as against Fentanyl with Ketamine combination.

\section{Methodology}

After institutional scientific and ethical committee approval, a written informed consent was obtained from parents. 70 paediatric patients, American Society of Anesthesiologists (ASA) physical status II and III, aged between 4 to 12 yrs with acyanotic congenital heart disease admitted for elective interventional cardiac catheterization procedures are included in this study. They are randomly allocated into two groups using random number generated by the computer.

In group 1 Dexmedetomidine-Ketamine (D) $(n=35)$ : Patients were given intravenous Dexmedetomidine $1 \mathrm{mcg} / \mathrm{kg}$ over $10 \mathrm{~min}$ before the procedure and a $1 \mathrm{mg} / \mathrm{kg}$ bolus of Ketamine.

In group 2 Fentanyl-Ketamine $(\mathrm{F})(\mathrm{n}=35)$ : Patients were given intravenous Fentanyl at a $1 \mathrm{mcg} / \mathrm{kg}$ over $10 \mathrm{~min}$ before the procedure and $1 \mathrm{mg} / \mathrm{kg}$ bolus of Ketamine.

\section{Study Design}

"A prospective, randomized, comparative study"

\section{Sampling Size and Technique}

Based on the previous study ${ }^{[13]}$ a sample size of 35 in each group would give the power of $90 \%$ and alpha error of 0.05

\section{Inclusion Criteria}

All patients aged between 4 to 12 years with acyanotic congenital heart disease scheduled for interventional cardiac catherization procedures.

\section{Exclusion Criteria}

- Children < 4 years in age and > 12 years in age;

- Children with chromosomal abnormalities or other multiple congenital anomalies, or hepatic or renal dysfunction.

- Children with cyanotic heart diseases

- Emergency procedures

- Patients requiring mechanical ventilation or intravenous inotropic support

\section{Results}

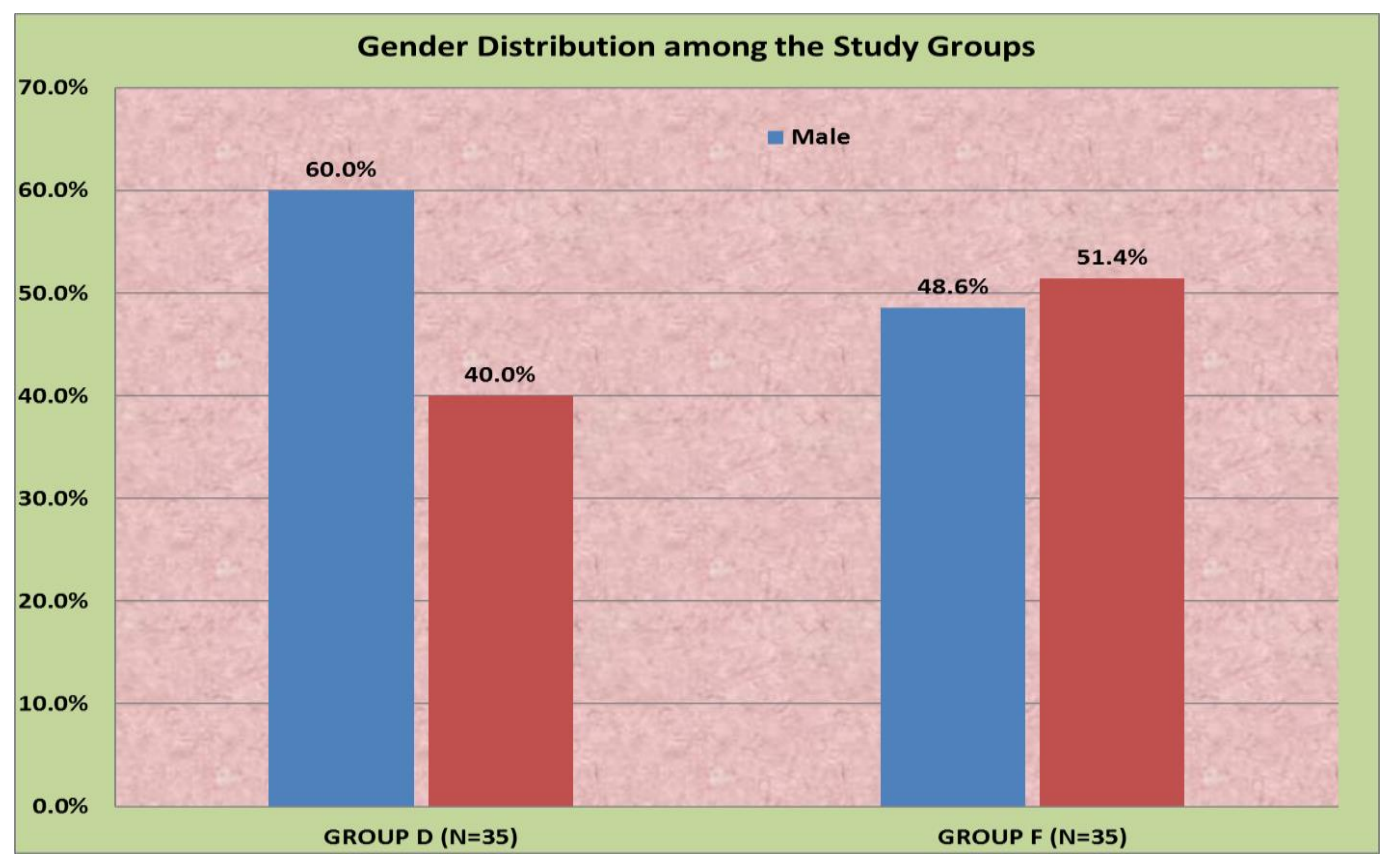

Graph 1: Gender Distribution 
The males in group D were 21 out of 35 patients and males in group $\mathrm{F}$ were 17 out of 35 .This distribution of gender in between two groups is not statistically significant $(\mathrm{p}>0.05)$.

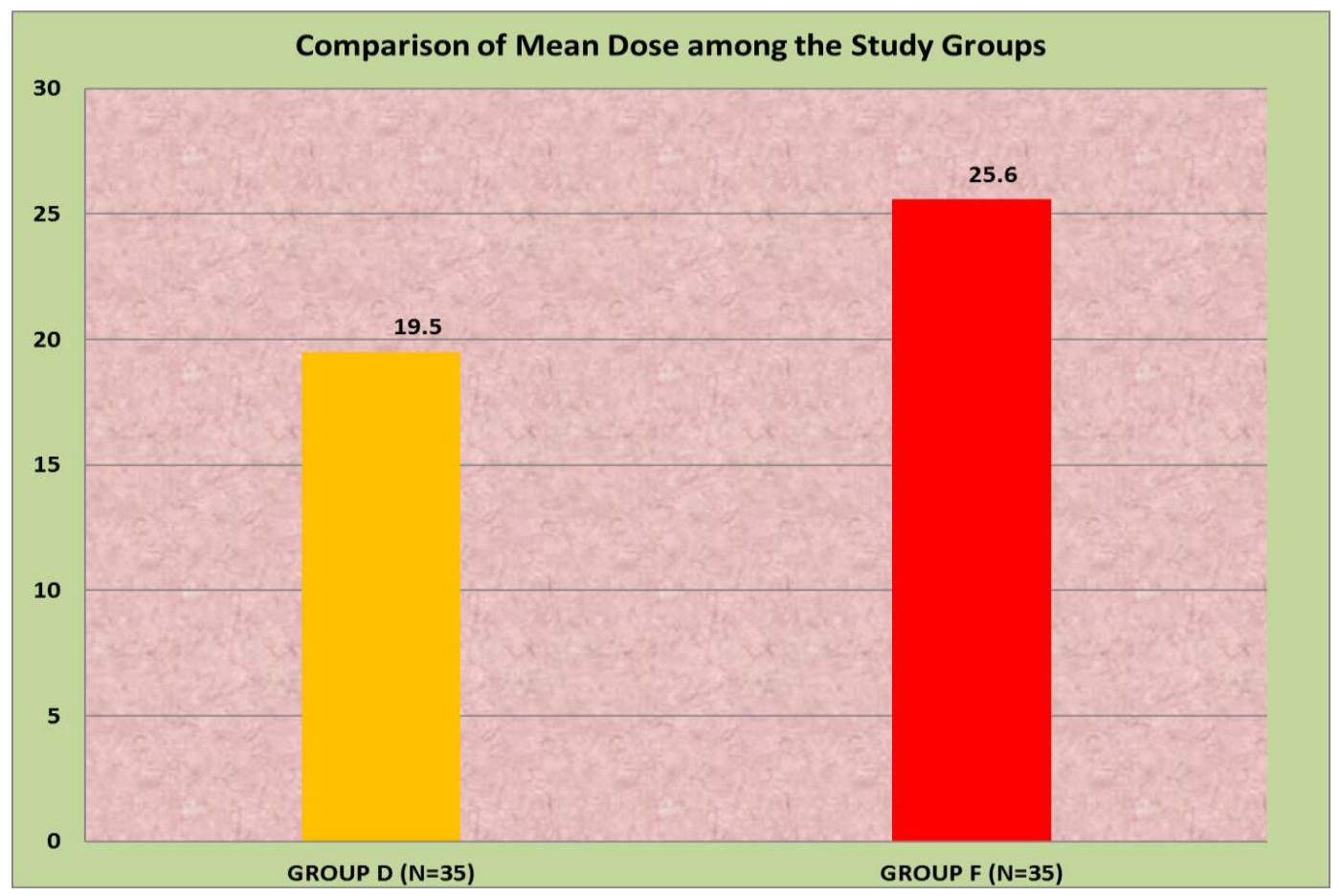

Graph 2: Dose of Test Drugs

The mean dose of test drugs either dexmedetomidine or fentanyl in their respective groups on comparison was significant which can be attributed to the difference in distribution of weight. [Table no 7 and graph no 3] $[P<0.05$ ] Used as indicator for achieving the adequate sedation. We used the score of 4 as attainment of adequate somnolescence with a dose of ketamine $1 \mathrm{mg} / \mathrm{kg}$ with test drug of $1 \mathrm{mcg} / \mathrm{kg}$ of dexmedetomidine in group $\mathrm{D}$ and fentanyl in group $\mathrm{F}$. As and when required we also supplemented the additional ketamine $0.5 \mathrm{mg} / \mathrm{kg}$ to attain the Ramsay score of 4 .

Table 1: Ramsay Score Distribution among the Groups

\begin{tabular}{|c|c|c|c|c|c|}
\hline & \multicolumn{2}{|c|}{ Ramsay Score } & \multirow{2}{*}{ Total } & \multirow{2}{*}{$\chi^{\mathbf{2}}$ value $^{*}$} & \multirow{2}{*}{ P value } \\
\cline { 2 - 4 } Group D & 3 & 4 & 35 & & \\
\cline { 2 - 4 } & 1 & 34 & $100.0 \%$ & \multirow{2}{*}{17.481} & $<0.001$ \\
\hline \multirow{2}{*}{ Group F } & $2.9 \%$ & $97.1 \%$ & 35 & \\
\cline { 2 - 4 } & $45.7 \%$ & $54.3 \%$ & $100.0 \%$ & \\
\cline { 2 - 4 } Total & 17 & 53 & 70 & & \\
\cline { 2 - 4 } & $24.3 \%$ & $75.7 \%$ & $100.0 \%$ & & \\
\hline
\end{tabular}

The graph clearly shows that in group D the percentage of patients who attained Ramsay score of 3 was $2.9 \%$ while in group f were $45.7 \%$ and this difference in Ramsay score is statiscally significant with a $\mathrm{P}$ value $<0.05$.

Table 2: Response Score Distribution

\begin{tabular}{|c|c|c|c|c|c|c|}
\hline & \multicolumn{3}{|c|}{ Response Score } & \multirow{2}{*}{ Total } & \multirow{2}{*}{$\chi^{\mathbf{2}}$ value $^{*}$} & \multirow{2}{*}{ P value } \\
\cline { 2 - 5 } & Marked & Moderate & Minimal/No & & \\
\multirow{2}{*}{ GROUP D } & 0 & 0 & 35 & 35 & \\
\cline { 2 - 5 } & $.0 \%$ & $.0 \%$ & $100.0 \%$ & $100.0 \%$ & & \\
\multirow{2}{*}{ GROUP F } & 2 & 12 & 21 & 35 & \multirow{2}{*}{17.500} & \multirow{2}{*}{$<0.001$} \\
\cline { 2 - 5 } & $5.7 \%$ & $34.3 \%$ & $60.0 \%$ & $100.0 \%$ & & \\
\cline { 2 - 5 } Total & 2 & 12 & 56 & 70 & & \\
\cline { 2 - 5 } & $2.9 \%$ & $17.1 \%$ & $80.0 \%$ & $100.0 \%$ & & \\
\hline
\end{tabular}

This graph and chart show the response of child to the infiltration of local anesthetic and femoral puncture. The Three Tier Response score was used to assess the response. It was graded as marked (1), moderate (2), minimal or no response (3)
In group $\mathrm{D}$, patients had minimal or no response to the local infiltration but in group $\mathrm{F} 34.3 \%$ had moderate response and $5.7 \%$ had marked response which was clinically as well as the statistically significant. 


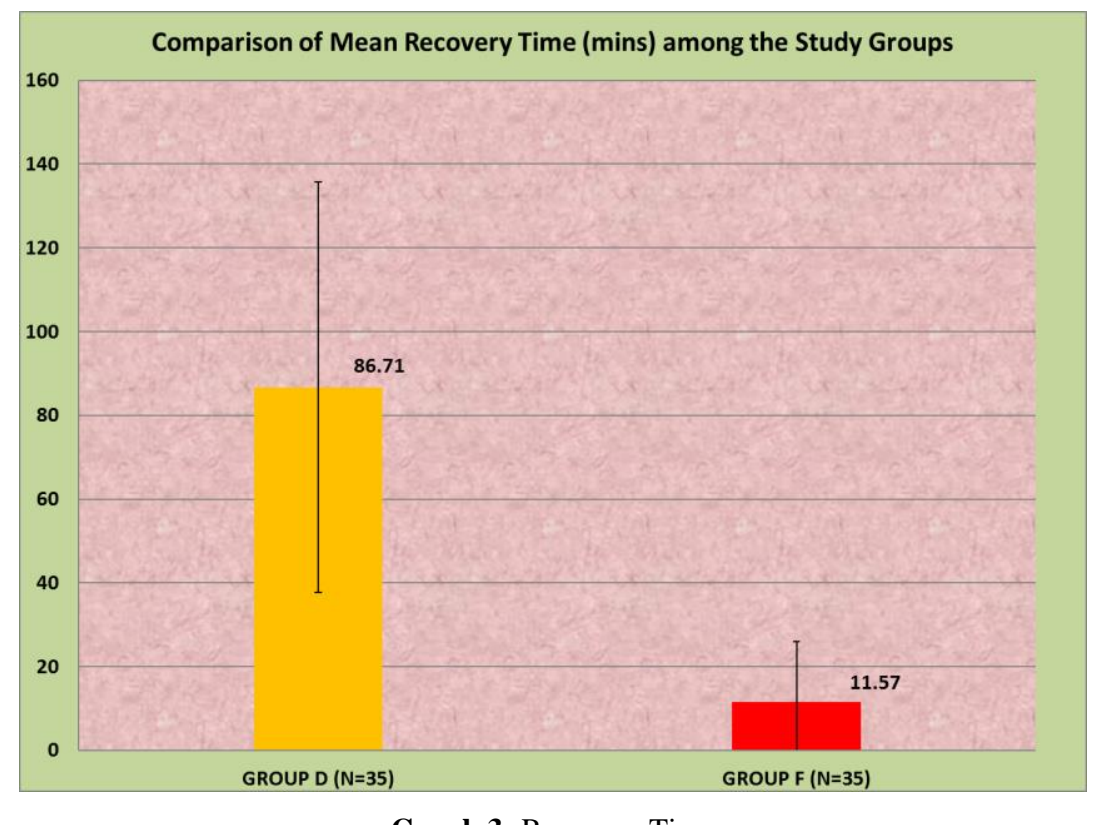

Graph 3: Recovery Time

The graph and chart clearly indicate that the recovery time which was time between the last dose of ketamine and attainment of steward score of 7 , a calm co-operative awake child moving all four limbs. The recovery time in group D on an average was $86.71 \pm 49.019$ mins and in group $F$ was $11.57 \pm 14.353 \mathrm{mins}$ this was statistically significant and clinically as well.

Out of 35 patients in the group F 13 children were awake on table .excluding them also the there is a significant difference in recovery time
However in group D 22 patients did not receive any additional ketamine the first dose of ketamine used for induction became the last dose also. Thus the above mentioned recovery time included duration of procedure in those 22 patients of group D. hence we defined sedation time and recovery time.

It's the time from the start of procedure to the attainment of steward score of 7 in CCU. its the total duration of sedation achieved by these drug combinations.

Table 3: Sedation Time

\begin{tabular}{|c|c|c|c|c|c|c|c|c|}
\hline GROUP & N & Mean & SD & Median & Min. & Max. & Mann-whitney U* & P value \\
\cline { 1 - 6 } Drug D & 35 & 98.7 & 48.101 & 90.00 & 20 & 205 & \multirow{2}{*}{102.500} & \multirow{2}{*}{$<0.001$} \\
\hline Drug F & 35 & 44.1 & 20.083 & 35.00 & 15 & 95 & & \\
\hline
\end{tabular}

There is significant difference in the mean duration of sedation attained by group D and group F both statistically and clinically. The sedation time does include the procedure duration and duration from end of procedure to attainment of the steward score of 7 .
This difference in sedation time can be attributed to the recovery time difference as the mean procedure time [CCL TIME] is not statistically and clinically significant between the two groups.

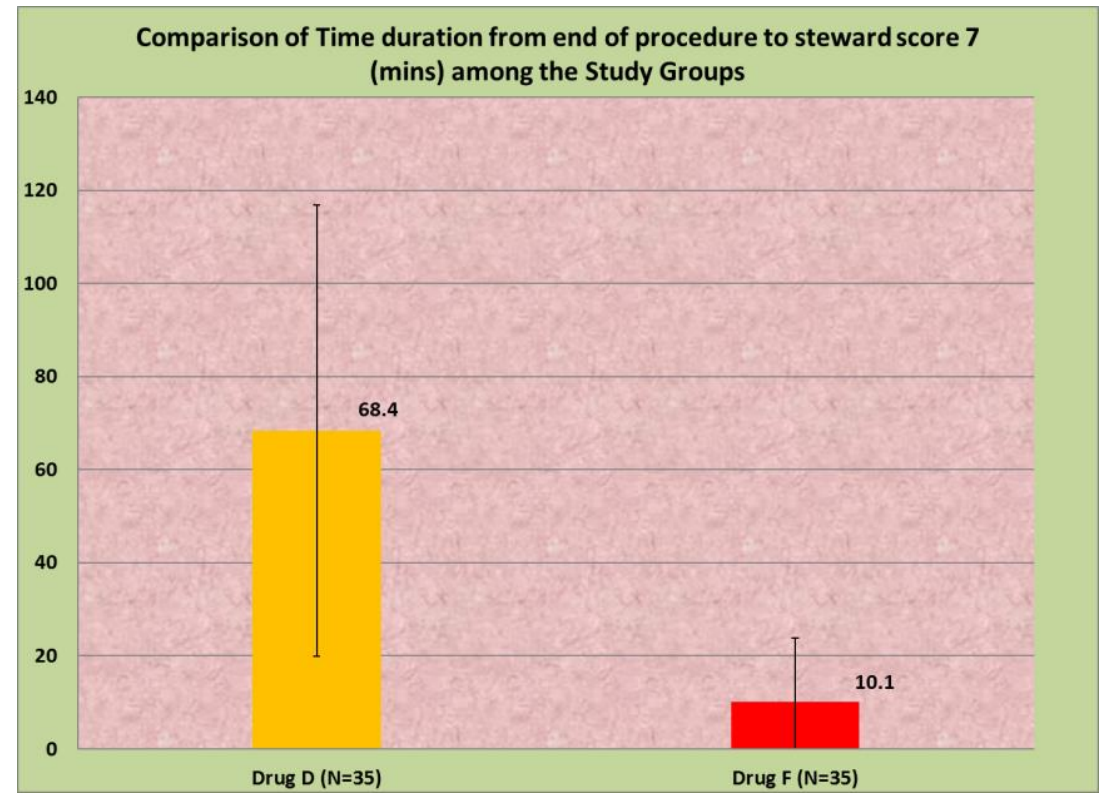

Graph 4: Recovery Time 
The graph and chart show the mean time elapsed between the end of procedure and attainment of steward score of 7 in CCU. This is statistically and clinical significant. This duration is excluding the procedure time.

\section{Discussion}

Sedation in catherisation lab has been adventurous and exploring arena. With complicated cases being done with minimal access, the anesthesiologist has a prime role to play in success of the procedure as well as the safety of the patient.

Multiple drugs as a sole agent or in combination have been tried and reported. The mild to moderate sedation to deep sedation and general anesthesia are in a continuum .There is a delicate balance exists between general anesthesia and deep sedation.

In our study 70 children [ 35 in each group d and group F] aged between $4-12 y r s$ belonging to ASA class II,III undergoing elective cardiac catherisation procedures like device closures of congenital heart defects were sedated with ketamine and study drug i.e dexmedetomidine in group $\mathrm{D}$ and fentanyl in group $\mathrm{F}$.

To maintain the sedation \& analgesia, our study showed that the total dose of ketamine required in group D was less as compared to Group F. [1.08 $\pm 0.293 \mathrm{vs} 1.80 \pm 0.644 \mathrm{mg} / \mathrm{kg}]$.

Mester and collaegues ${ }^{7}$ studied retrospectively the dexmedetomidine sedation in CCL. In the study population of 16 , only 3 required the additional ketamine to supplement the sedation apart from the bolus dose of ketamine at induction.

Similar to our study, Ashgan Raouf Ali and Mohamed N. El Ghoneimy ${ }^{[8]}$ reported that propofol/ dexmedetomidine combination was accompanied with less propofol consumption, prolonged analgesia and lower incidence of intra-procedural and post-procedural complications in children posted for elective ESWL.

Munro and others [9] described their experience with dexmedetomidine in children in CCL and reported that in dexmedetomidine group $12 / 20$ patients were given a propofol bolus at some time during the procedure because of movement, increasing BIS value or in anticipation of stimulation

We aimed at attaining the Ramsay score of 4 to ensure adequate sedation for local anaesthetic infiltration at femoral puncture site in our study subjects.

Our study showed that the difference in acheiving a Ramsay score of 4 between the two groups were significant $[\mathrm{p}=0.001]$. This implied to us that the dexmedetomidine and fentanyl though both are given over ten mins as a bolus along with ketamine $1 \mathrm{mg} / \mathrm{kg}$ for the attainment of depth of sedation was better with dexmedetomidine ketamine combination than with fentanyl and ketamine combination.

Tosun and co-authors ${ }^{[10]}$ compared the combination of dexmedetomidine with ketamine and propfol with ketamine for sedation in spontaneous breathing patients in cath lab.They also used Ramsay scores to compare the sedation between the groups. They did not find any significant difference in sedation scores however higher sedation scores were found at 30, 45, and 60 minutes than at baseline $(\mathrm{p}<0.05)$ in dexmedetomidine and ketamine group compared to in propofol with ketamine, sedation scores were higher at 15, 30, 45, and 60 minutes. Which implied to us that the dexmedetomidine and ketamine combination had slower onset of sedation.
In another study, Assad and others [11] compared dexmedetomidine and ketamine (DK) with fentanyl ketamine(FK) combination for sedation in extracorporeal shock wave lithotripsy. They compared sedation using modified observers assessment of alertness and awake fullness score. There was no significant difference between the two groups throughout the procedure while there was a significant increase in sedation score after 40, 45, $50 \mathrm{~min}$ (recovery period) in FK group in comparison with DK group.

In a third study by Berkenbosch ${ }^{[12]}$ and his team evaluated the effectiveness of dexmedetomidine in comparison with the conventional sedative techniques of chloral hydrate and midazolam. They compared the effectiveness of sedation using modification of the University of Michigan Sedation Scale. They achieved the sedation score of 3 and 4 prior to initiation of any non-invasive procedure. 15 children who failed sedation with chloral hydrate or midazolam or both midazolam were effectively sedated with dexmdetomidine The three tier scoring system was used in our study to assess the response to local anesthesia infiltration.

The response score indicated us that depth of sedation was maintained throughout the procedure. In group $\mathrm{D}$, patients had minimal or no response to the local infiltration but in group F, 34.7\% of patients had moderate response and $2.3 \%$ of patients had marked response which was clinically as well as the statistically significant $[p=0.001]$.

Whereas Assad and his team ${ }^{[1]}$ analyzed this combination of dexmedetomidine and ketamine with fentanyl and ketamine $[\mathrm{FK}]$ in terms of BIS scores in ESWL procedures. There was no significant difference in BIS scores between the two groups throughout the procedure, while there was a significant increase in FK group during the postoperative period 40, 45 and $50 \mathrm{~min}$

In spontaneously breathing children in cath lab, BIS values were monitored for the depth of sedation by Tosun and others ${ }^{10}$, wherein 2 groups were monitored. Group1 subjects received dexmedetomidine and ketamine and Group2 subjects received propofol and ketamine. They found that the BIS values in the Group2 at 15 and 30 minutes were significantly lower than the first group; BIS values were lower at 15, 30, 45, and 60 minutes than at baseline in both the groups.

In children undergoing elective ESWL procedures, Ashgan Raouf Ali and Mohamed N. El Ghoneimy ${ }^{[13]}$ compared the dexmedetomidine with fentanyl as an adjunct to propofol. They used the BIS values to assess the depth of sedation in both the groups. Comparing the two groups, there was no significant difference in BIS values at all-time intervals.

The recovery time was defined ${ }^{[14]}$ as the time from the last dose of ketamine to achievement of Steward score of 7; i.e child is awake, able to cough on command \& moving all the four limbs This was assessed in CCU post procedure and the duration was noted in mins. In the current study it was observed the recovery time in group D was at an average of $86.71 \pm 49.019$ mins and in group $\mathrm{F}$, the average was $11.57 \pm 17.353 \mathrm{mins}$

However in group D 22 patients did not receive any additional ketamine the first dose of ketamine used for induction became the last dose also. Thus the above mentioned recovery time included duration of procedure in those 22 patients of group Hence we defined sedation time and recovery time 


\section{Conclusion}

The patients in dexmedetomidine and ketamine combination had better Ramsay score of sedation, better response score and required lesser supplemental dose of ketamine. However they had prolonged recovery period as compared to the fentanyl ketamine group patients.

\section{References}

1. Gross LW. Non-operating Room anaesthesia In: Miller RD, Cohen HN, Eriksson IL, Weiner-Kronish PJ, Young LW. Miller's Anaesthesia. $8^{\mathrm{TH}}$ edition. Philadeplphia: Elsevier 2015, 2646-73.

2. Youn AM, Ko YK, Kim YH. Anesthesia and sedation outside of the operating room. Korean journal of anesthesiology 2015;68(4):323-31.

3. ASo A. Statement on Nonoperating Room Anesthetizing Locations 2013. Accessed 2/12/2015. Reference Source

4. Mueller RL, Sanborn TA. The history of interventional cardiology: cardiac catheterization, angioplasty, and related interventions. American heart journal 1995;129(1):146-72.

5. Bourassa, Martial. The history of cardiac catheterization. Can J Cardiol 2005;21(12):1011-4.

6. Abbas SM, Rashid A, Latif H. Sedation for children undergoing cardiac catheterization: A review of literature. Journal of the Pakistan Medical Association 2012;62(2):159.

7. Mester R, Easley RB, Brady KM, Chilson K, Tobias JD. Monitored anesthesia care with a combination of ketamine and dexmedetomidine during cardiac catheterization. American journal of therapeutics 2008;15(1):24-30.

8. Ramsay M, Savege TM, Simpson BR, Goodwin R. Controlled sedation with alphaxalone- alphadolone. $\mathrm{Br}$ Med J 1974;2(5920):656-9.

9. Munro HM, Tirotta CF, Felix DE, Lagueruela RG, Madril DR, Zahn EM et al. Initial experience with Dexmedetomidine for diagnostic and interventional cardiac catheterization in children: Pediatric Anesthesia 2007; 17(2):109-12.

10. Tosun Z, Akin A, Guler G, Esmaoglu A, Boyaci A. Dexmedetomidine-ketamine and propofol-ketamine combinations for anesthesia in spontaneously breathing pediatric patients undergoing cardiac catheterization. Journal of cardiothoracic and vascular anesthesia 2006;20(4):515-9.

11. Assad OM. Comparative study between dexmedetomidine-ketamine and fentanyl-ketamine combinations for sedation in patients undergoing extracorporeal shock wave lithotripsy. A randomized double blinded study. Egyptian Journal of Anaesthesia. $2015 ; 31(1): 35-41$.

12. Berkenbosch JW, Wankum PC, Tobias JD. Prospective evaluation of dexmedetomidine for noninvasive procedural sedation in children. Pediatric Critical Care Medicine 2005;6(4):435-9.

13. Ali AR, El Ghoneimy MN. Dexmedetomidine versus fentanyl as adjuvant to propofol: comparative study in children undergoing extracorporeal shock wave lithotripsy. European Journal of Anaesthesiology 2010;27(12):1058-64.

14. Shukry M, Miller JA. Update on dexmedetomidine: use in nonintubated patients requiring sedation for surgical procedures. Therapeutics and clinical risk management 2010;6:111. 